\title{
Experimental assessment of regenerative properties of platelet rich plasma on the human skin - a review
}

\author{
Marco Mario Tresoldi ${ }^{1,2,3}$, Angela Faga ${ }^{3}$, Giovanni Nicoletti ${ }^{1,3,4}$ \\ 'Unit of Plastic and Reconstructive Surgery, Department of Clinical Surgical, Diagnostic and Pediatric Sciences, University of \\ Pavia, Pavia 27100, Italy. \\ ${ }^{2}$ Department of Surgery, Istituti Clinici Scientifici Maugeri, Pavia 27100, Italy. \\ ${ }^{3}$ Department of Clinical Surgical, Diagnostic and Pediatric Sciences and Department of Public Health and Experimental and \\ Forensic Medicine, Advanced Technologies for Regenerative Medicine and Inductive Surgery Research Center, University of \\ Pavia, Pavia 27100, Italy. \\ ${ }^{4}$ Plastic Surgery Unit, Azienda Socio-Sanitaria Territoriale di Pavia, Pavia 27100, Italy.
}

Correspondence to: Dr. Marco Mario Tresoldi, Unit of Plastic and Reconstructive Surgery, Department of Clinical Surgical Diagnostic and Pediatric Sciences, University of Pavia, Viale Brambilla, 74, Pavia 27100, Italy.

E-mail: marcomario.tresoldi@unipv.it

\begin{abstract}
How to cite this article: Tresoldi MM, Faga A, Nicoletti G. Experimental assessment of regenerative properties of platelet rich
\end{abstract} plasma on the human skin - a review. Plast Aesthet Res 2022;9:12. https://dx.doi.org/10.20517/2347-9264.2021.85

Received: 27 Jul 2021 First Decision: 29 Nov 2021 Revised: 13 Dec 2021 Accepted: 22 Dec 2021 Published: 8 Feb 2022

Academic Editors: Pietro Gentile, Wen-Guo Cui Copy Editor: Xi-Jun Chen Production Editor: Xi-Jun Chen

\begin{abstract}
Several studies demonstrated the favorable effects of platelet rich plasma (PRP) on the skin and promoted its wide use in clinical practice. The growth factors stored in platelet alfa-granules allow for the tissue regeneration and the main fields of application of PRP in current clinical practice are the cartilage and musculoskeletal defects, osteoarthritis and other bone disorders, chronic and difficult to heal wounds, and aesthetic procedures. The relevant number of different PRP preparation protocols may explain the inconsistency of the different clinical outcomes reported in the literature. Despite the technological advances in PRP preparation, the objective assessment of the clinical efficacy of PRP from the literature reports still is difficult due to the low homogeneity of the samples in terms of both inclusion criteria and size. Therefore, it might be useful to establish standardized and reproducible experimental models to confirm and objectively measure the effectiveness of the available clinical results. Many experimental investigations have been carried out to objectively assess the effectiveness of PRP and platelet gel on several tissues. As far as the skin is concerned, the studies carried out to date are limited to fibroblasts in in-vitro culture models or to collagen, vascular supply, epithelium, and hair follicle in in-vivo models. The skin, however, is a very complex organ, where different cell lines coexist and feature complex mutual interaction. A model that combines the advantages of both in-vitro and in-vivo cultures is the ex-vivo model. The
\end{abstract}

The Author(s) 2022. Open Access This article is licensed under a Creative Commons Attribution 4.0 International License (https://creativecommons.org/licenses/by/4.0/), which permits unrestricted use, sharing, adaptation, distribution and reproduction in any medium or format, for any purpose, even commercially, as long as you give appropriate credit to the original author(s) and the source, provide a link to the Creative Commons license, and indicate if changes were made. 
demonstration of the platelet derived growth factors effects through the ex-vivo human full-thickness skin culture model is a keystone to support the evidence of the PRP effectiveness, as it represents an objective, fast, reproducible, and ethical investigational method.

Keywords: Platelet rich plasma, PRP, ex-vivo, regeneration, culture techniques, skin

\section{INTRODUCTION}

Several studies demonstrated the favorable action of platelet rich plasma (PRP) on human tissues and promoted its wide use in clinical practice ${ }^{[1]}$. The growth factors stored in platelet alfa-granules allow for the tissue regeneration and the main fields of application of PRP in current clinical practice are the wound healing process, cartilage and musculoskeletal defects, osteoarthritis and other bone disorders, chronic and difficult to heal wounds, and aesthetic procedures in an increasing number of medical specialties ${ }^{[2-11]}$. Nevertheless, the reported outcomes are still inconsistent.

The properties of PRP may be affected by the patients' age, gender, body mass index, diet, comorbidities, healing ability, and lifestyle $\mathrm{e}^{[12,13]}$.

The variables affecting the clinical results may be related also to the different modalities of PRP production starting from the whole human blood, the number of spin cycles related to the g-force and time in the centrifugation process, the platelet amount and concentration and the type of growth factor obtained ${ }^{[1,15]}$.

Currently, there are more than 40 kits on the market to produce platelet-rich substance from a whole blood sample. The kits differ from each other in the number and capacity of the tubes used for processing, the "opened" or "closed" system to transfer the blood into the tubes and obtain the suspension, the blood separation system, and the number of steps required to obtain the $\mathrm{PRP}^{[16]}$.

Such a large number of preparation methods may explain the inconsistency of the results reported by different authors ${ }^{[17]}$. Among these kits, the most reliable ones are closed systems, which limit operator errors and reduce the risk of microbial contamination, avoiding blood transfers via syringes between the preparation steps ${ }^{[18,19]}$.

According to the closed modality, the PRP samples feature a fixed concentration of specific biomolecules.

Despite these technological advances in PRP preparation, the objective assessment of the clinical efficacy of PRP from the literature reports still is difficult due to the low homogeneity of the samples in terms of both inclusion criteria (e.g., age, pathologies, drug therapy, and severity of the defect to be treated), and depending on the field of application such as dermatology, plastic surgery, or orthopedics; goals can vary from improvement of skin quality to regeneration of hair loss to cartilage or bone regeneration. Therefore, it may be useful to establish standardized and reproducible experimental models to confirm and objectively measure the effectiveness of the currently available clinical results.

\section{EXPERIMENTAL MODELS}

The above-mentioned aim still is difficult to achieve due to the extreme variability and different limits of the experimental models, both in-vitro and in-vivo. Many experimental investigations have been carried out to objectively assess the effectiveness of PRP and platelet gel on muscle, bone, cartilage, and endometrium ${ }^{[20-24]}$. 
As far as the skin is concerned, the studies carried out to date are limited to fibroblasts in in-vitro culture models or to collagen, vascular supply, epithelium and hair follicle in in-vivo models ${ }^{[25]}$.

The skin, however, is very complex an organ, where different cell lines coexist and feature very complex mutual interaction ${ }^{[26]}$.

Different experimental models with skin derived cells and/or structures are reported for both pharmacological and dermatological investigations, but most of them lack a fundamental feature, the ability to reproduce results on physiological skin in the in-vivo environment ${ }^{[27,28]}$.

In-vitro models are very popular as they are cheap and easy to use, and they usually include one or more skin cell types. The latter setting is termed co-culture and such a model allows for the observation of the mutual cell interactions. Usually, skin co-cultures may include keratinocytes and fibroblasts, keratinocytes and melanocytes, or keratinocytes and neurons. However, these models fail to reproduce the in-vivo threedimensional tissue morphology and physiology ${ }^{[29-31]}$.

In-vivo models are animal or human. The animal in-vivo model allows studies and observations of pharmacological molecules and compounds directly on the living animal or after sacrificing it. Thanks to this model many data regarding regeneration, re-epithelialization, neo-angiogenesis, and innervation have been obtained ${ }^{[32]}$.

However, both ethical and high costs issues significantly limit this model.

Moreover, the regenerative potential in the animals is significantly different from that of humans ${ }^{[33]}$.

Therefore, it is a common trend in the scientific world to search for alternative approaches to experimenting in living animals.

The human in-vivo model is also used to test pharmacological molecules and compounds especially in patients suffering from dermatological diseases, whilst cosmetics can be tested on healthy volunteers. This is supposed to be the ideal model, although it is rarely used due to high costs and relevant ethical inquiries ${ }^{[28]}$.

For the previously reported reasons and for the complexity of biochemical and biophysical processes involved in tissue regeneration, computational models were developed to understand cell growth and to device new scaffolds and tissue replacements. Two computational models have been developed so far: the in-silico and in-virtuo models ${ }^{[27,34]}$.

The in-silico model seems very promising both alone and in combination with other models ${ }^{[27]}$.

Given the high complexity of the biochemical and biophysical processes involved in wound healing, the simulation offered by the in-silico model represents a powerful tool in understanding the behaviour of distinct cell types, growth factors, and the extracellular matrix within the human skin structure.

However, this model does not yet reproduce the physical features of the whole human $\operatorname{skin}^{[3,356]}$. 
The in-virtuo model is based upon powerful software gathering all known data about cellular interactions, gene expression, and vitality, but to date such a model is still lacking enough data to be considered a valid alternative to the in-vivo models ${ }^{[34]}$.

A model that combines the advantages of both in-vitro and in-vivo cultures is the ex-vivo model. It is also called an organotypic model because it maintains the skin's three-dimensional structure and best reproduces the tissue's physiology. In this culture model, the skin can be both laboratory-engineered and native after harvest from living individuals.

The tissue-engineered skin - also known as reconstructed skin or reconstituted skin - is composed of two separate components, the epidermis and dermis. The epidermis is made by keratinocytes obtained from a skin explant and cultured on a support - for example, collagen - with the appropriate medium allowing for the appropriate chemical-physical environment ${ }^{[37]}$. During this period, keratinocytes progressively colonise the support and create a multi-layered, three-dimensional epithelial tissue. The dermis, instead, is made of different extra-cellular matrix equivalents which can be both natural and synthetic ${ }^{[37]}$. The first reported tissue-engineered skin model included only two cell types, keratinocytes and fibroblasts derived from native skin explants ${ }^{[37]}$. Subsequently, other cell types, such as melanocytes, endothelial cells, Langerhans cells, nerve cells, and adipocytes, enriched these models ${ }^{[37]}$.

The ex-vivo native human skin obtained through surgical resection in skin-reducing procedures in healthy individuals is an effective experimental model to investigate the effects of topically applied products ${ }^{[3]]}$. The explanted skin can be used both as a whole or as a single layer after separating the epidermis from the dermis. The whole ex-vivo skin model keeps all skin cell types - keratinocytes, fibroblasts, melanocytes, Langerhans cells, endothelial cells, and Merkel cells - and it is only lacking in vascularisation and innervation ${ }^{[28]}$.

As this experimental model maintains the structure of the native human skin, including the complete cell population and the dermal matrix components (i.e., collagen, elastin, glycosaminoglycans, etc.); it most closely approximates the effects of substances on in-vivo human $\operatorname{skin}^{[28,38]}$.

It may allow for the evaluation of the effects of topical products on human skin, either in the cosmetic or in the pharmaceutical context ${ }^{[28]}$.

This model might also contribute to further disclose the fine mechanisms of skin regeneration.

An original ex-vivo PRP enriched human skin experimental model was recently devised by Nicoletti et al. ${ }^{[39]}$. In this model, human skin samples were derived from surgical specimens harvested during breast reduction procedures. Prior to culture, the samples were wounded in their central portion in order to create a standard skin lesion. The tissue cultures were carried out under three different conditions:

- Enriched Dubecco's Modified Eagle Medium (DMEM) with saline solution in the central wound (control);

- Enriched DMEM with the same medium in the central wound;

- Enriched DMEM plus 2.5\% autologous PRP, with the same PRP added medium in the central wound. 
Morphological analysis was carried out at $0 \mathrm{~h}$ (To) and on days 1, 3, 5, and 10 (T1-T3-T5-T10) using hematoxylin and eosin, Masson's trichrome staining, Weigert staining, and Ki-67 staining to identify the skin morphological and functional features in the different experimental conditions.

Therefore, the step by step histological and immunohistochemical regenerative effects of PRP on human skin wound repair and regeneration process was observed in detail over a period of 10 days. The overall vitality of the cultured skin samples was enhanced by the addition of PRP, as demonstrated by the macroscopic persistence of a lively pink color, up to, and even until 10 days, while the samples, cultured in the other conditions displayed a shift toward a yellow cadaveric color well before 5 days. A favorable modulation of the epithelial cells and fibroblasts proliferation was demonstrated by a regular reepithelialization of the experimental wound. A relevant anti-inflammatory action was demonstrated by the inhibition of lymph-plasma-cells infiltration ${ }^{[39]}$.

PRP also allowed for an inhibitory effect on both the collagen and elastic fibers' de-structuration and a favorable modulation of the re-organization of these fibers; in fact, the samples cultured with the PRP added medium displayed a regular and compact dermal pattern of prevalent thin collagen fibers in a shorter time compared to the other experimental conditions.

This original experimental ex-vivo PRP enriched skin culture model allowed for the step-by-step objective demonstration of the effects of platelet derived growth factors on the wound healing process. For the first time it was possible to observe and directly describe the stimulating action of platelet derived growth factors on the proliferation of keratinocytes, proliferation of fibroblasts, and synthesis of collagen, as well as the anti-inflammatory effects. This kind of model can explain in detail the main contribution of PRP in the field of human skin regeneration and it is possible to apply the ex-vivo model to many other tissues to observe and measure the efficacy of PRP in achieving the proposed objectives.

\section{CONCLUSIONS}

The demonstration of the platelet derived growth factors effects through the ex-vivo human full-thickness skin culture model is a keystone to support the evidence of PRP's effectiveness, as it represents an objective, fast, reproducible, and ethical investigation method. Similarly, the use of PRP on other types of human tissues with the ex-vivo model can demonstrate its property in tissue regeneration.

\section{DECLARATIONS}

\section{Authors' contributions}

Made substantial contributions to conception and writing of the review and performed the literature analysis and interpretation: Tresoldi MM, Faga A

Made substantial contributions to writing of the review: Nicoletti G

\section{Availability of data and materials}

Not applicable.

\section{Financial support and sponsorship}

None.

\section{Conflicts of interest}

All authors declared that there are no conflicts of interest. 


\section{Ethical approval and consent to participate}

Not applicable.

\section{Consent for publication}

Not applicable.

\section{Copyright}

(C) The Author(s) 2022.

\section{REFERENCES}

1. Samadi P, Sheykhhasan M, Khoshinani HM. The use of platelet-rich plasma in aesthetic and regenerative medicine: a comprehensive review. Aesthetic Plast Surg 2019;43:803-14. DOI PubMed

2. Gentile P, Scioli MG, Bielli A, Orlandi A, Cervelli V. Concise review: the use of adipose-derived stromal vascular fraction cells and platelet rich plasma in regenerative plastic surgery. Stem Cells 2017;35:117-34. DOI PubMed

3. Bottegoni C, Dei Giudici L, Salvemini S, Chiurazzi E, Bencivenga R, Gigante A. Homologous platelet-rich plasma for the treatment of knee osteoarthritis in selected elderly patients: an open-label, uncontrolled, pilot study. Ther Adv Musculoskelet Dis 2016;8:35-41. DOI PubMed PMC

4. Stessuk T, Puzzi MB, Chaim EA, et al. Platelet-rich plasma (PRP) and adipose-derived mesenchymal stem cells: stimulatory effects on proliferation and migration of fibroblasts and keratinocytes in vitro. Arch Dermatol Res 2016;308:511-20. DOI PubMed

5. Picard F, Hersant B, La Padula S, Meningaud JP. Platelet-rich plasma-enriched autologous fat graft in regenerative and aesthetic facial surgery: technical note. J Stomatol Oral Maxillofac Surg 2017;118:228-31. DOI PubMed

6. Hersant B, Sidahmed-mezi M, Lapadula S, Niddam J, Bouhassira J, Meningaud JP. Efficacy of autologous platelet-rich plasma glue in weight loss sequelae surgery and breast reduction: a prospective study. Plast Reconstr Surg Glob Open 2016;4:e871. DOI PubMed PMC

7. Sasaki GH. Micro-needling depth penetration, presence of pigment particles, and fluorescein-stained platelets: clinical usage for aesthetic concerns. Aesthet Surg J 2017;37:71-83. DOI PubMed

8. Jain NK, Gulati M. Platelet-rich plasma: a healing virtuoso. Blood Res 2016;51:3-5. DOI PubMed PMC

9. Everts P, Onishi K, Jayaram P, Lana JF, Mautner K. Platelet-rich plasma: new performance understandings and therapeutic considerations in 2020. Int J Mol Sci 2020;21:7794. DOI PubMed PMC

10. Etulain J. Platelets in wound healing and regenerative medicine. Platelets 2018;29:556-68. DOI PubMed

11. Maisel-Campbell AL, Ismail A, Reynolds KA, et al. A systematic review of the safety and effectiveness of platelet-rich plasma (PRP) for skin aging. Arch Dermatol Res 2020;312:301-15. DOI PubMed

12. Evanson JR, Guyton MK, Oliver DL, et al. Gender and age differences in growth factor concentrations from platelet-rich plasma in adults. Mil Med 2014;179:799-805. DOI PubMed

13. Miller CH, Rice AS, Garrett K, Stein SF. Gender, race and diet affect platelet function tests in normal subjects, contributing to a high rate of abnormal results. Br J Haematol 2014;165:842-53. DOI PubMed PMC

14. Anitua E, Sánchez M, Orive G, Andía I. The potential impact of the preparation rich in growth factors (PRGF) in different medical fields. Biomaterials 2007;28:4551-60. DOI PubMed

15. Castillo TN, Pouliot MA, Kim HJ, Dragoo JL. Comparison of growth factor and platelet concentration from commercial platelet-rich plasma separation systems. Am J Sports Med 2011;39:266-71. DOI PubMed

16. Gentile P, Calabrese C, De Angelis B, et al. Impact of the different preparation methods to obtain autologous non-activated plateletrich plasma (A-PRP) and activated platelet-rich plasma (AA-PRP) in plastic surgery: wound healing and hair regrowth evaluation. Int J Mol Sci 2020;21:431. DOI PubMed PMC

17. Kaux JF, Le Goff C, Seidel L, et al. [Comparative study of five techniques of preparation of platelet-rich plasma]. Pathol Biol (Paris) 2011;59:157-60. DOI PubMed

18. Mussano F, Genova T, Munaron L, Petrillo S, Erovigni F, Carossa S. Cytokine, chemokine, and growth factor profile of platelet-rich plasma. Platelets 2016;27:467-71. DOI PubMed

19. Oudelaar BW, Peerbooms JC, Huis In 't Veld R, Vochteloo AJH. Concentrations of blood components in commercial platelet-rich plasma separation systems: a review of the literature. Am J Sports Med 2019;47:479-87. DOI PubMed

20. Malhotra A, Pelletier MH, Yu Y, Walsh WR. Can platelet-rich plasma (PRP) improve bone healing? Arch Orthop Trauma Surg 2013;133:153-65. DOI PubMed

21. Laver L, Marom N, Dnyanesh L, Mei-Dan O, Espregueira-Mendes J, Gobbi A. PRP for degenerative cartilage disease: a systematic review of clinical studies. Cartilage 2017;8:341-64. DOI PubMed PMC

22. Liu F, Xu H, Huang H. A novel kartogenin-platelet-rich plasma gel enhances chondrogenesis of bone marrow mesenchymal stem cells in vitro and promotes wounded meniscus healing in vivo. Stem Cell Res Ther 2019;10:201. DOI PubMed PMC

23. Aghajanova L, Houshdaran S, Balayan S, et al. In vitro evidence that platelet-rich plasma stimulates cellular processes involved in endometrial regeneration. J Assist Reprod Genet 2018;35:757-70. DOI PubMed PMC

24. Beitzel K, McCarthy MB, Russell RP, Apostolakos J, Cote MP, Mazzocca AD. Learning about PRP using cell-based models. Muscles Ligaments Tendons J 2014;4:38-45. PubMed PMC 
25. Berndt S, Turzi A, Pittet-Cuénod B, Modarressi A. Autologous platelet-rich plasma (CuteCell PRP) safely boosts in vitro human fibroblast expansion. Tissue Eng Part A 2019;25:1550-63. DOI PubMed

26. Ruaro B, Soldano S, Smith V, et al. Correlation between circulating fibrocytes and dermal thickness in limited cutaneous systemic sclerosis patients: a pilot study. Rheumatol Int 2019;39:1369-76. DOI PubMed

27. Ud-Din S, Bayat A. Non-animal models of wound healing in cutaneous repair: In silico, in vitro, ex vivo, and in vivo models of wounds and scars in human skin. Wound Repair Regen 2017;25:164-76. DOI PubMed

28. Eberlin S, Silva MSD, Facchini G, et al. The ex vivo skin model as an alternative tool for the efficacy and safety evaluation of topical products. Altern Lab Anim 2020;48:10-22. DOI PubMed

29. Johnen C, Steffen I, Beichelt D, et al. Culture of subconfluent human fibroblasts and keratinocytes using biodegradable transfer membranes. Burns 2008;34:655-63. DOI PubMed

30. Lei TC, Virador VM, Vieira WD, Hearing VJ. A melanocyte-keratinocyte coculture model to assess regulators of pigmentation in vitro. Anal Biochem 2002;305:260-8. DOI PubMed

31. Château Y, Dorange G, Clément JF, et al. In vitro reconstruction of neuro-epidermal connections. J Invest Dermatol 2007;127:979-81. DOI PubMed

32. Faga A, Nicoletti G, Gregotti C, Finotti V, Nitto A, Gioglio L. Effects of thermal water on skin regeneration. Int J Mol Med 2012;29:732-40. DOI PubMed

33. Rippa AL, Kalabusheva EP, Vorotelyak EA. Regeneration of dermis: scarring and cells involved. Cells 2019;8:607. DOI PubMed PMC

34. Lebonvallet N, Jeanmaire C, Danoux L, Sibille P, Pauly G, Misery L. The evolution and use of skin explants: potential and limitations for dermatological research. Eur J Dermatol 2010;20:671-84. DOI PubMed

35. Pappalardo F, Russo G, Tshinanu FM, Viceconti M. In silico clinical trials: concepts and early adoptions. Brief Bioinform 2019;20:1699-708. DOI PubMed

36. Guerra A, Belinha J, Jorge RN. Modelling skin wound healing angiogenesis: a review. J Theor Biol 2018;459:1-17. DOI PubMed

37. Souto LR, Rehder J, Vassallo J, Cintra ML, Kraemer MH, Puzzi MB. Model for human skin reconstructed in vitro composed of associated dermis and epidermis. Sao Paulo Med J 2006;124:71-6. DOI PubMed

38. Nicoletti G, Saler M, Pellegatta T, et al. Ex vivo regenerative effects of a spring water. Biomed Rep 2017;7:508-14. DOI PubMed PMC

39. Nicoletti G, Saler M, Villani L, Rumolo A, Tresoldi MM, Faga A. Platelet rich plasma enhancement of skin regeneration in an ex-vivo human experimental model. Front Bioeng Biotechnol 2019;7:2. DOI PubMed PMC 